\title{
The treatment of magnetic buoyancy in flux transport dynamo models
}

\author{
Arnab Rai Choudhuria ${ }^{\mathrm{a}, *}$, Gopal Hazra ${ }^{\mathrm{a}, \mathrm{b}}$ \\ ${ }^{a}$ Department of Physics, Indian Institute of Science,Bangalore 560012, India \\ ${ }^{b}$ Indian Institute of Astrophysics, Bangalore-560034, India
}

\begin{abstract}
One important ingredient of flux transport dynamo models is the rise of the toroidal magnetic field through the convection zone due to magnetic buoyancy to produce bipolar sunspots and then the generation of the poloidal magnetic field from these bipolar sunspots due to the Babcock-Leighton mechanism. Over the years, two methods of treating magnetic buoyancy - a local method and a non-local method - have been used widely by different groups in constructing 2D kinematic models of the flux transport dynamo. We review both these methods and conclude that neither of them is fully satisfactory - presumably because magnetic buoyancy is an inherently 3D process. We also point out so far we do not have proper understanding of why sunspot emergence is restricted to rather low latitudes.
\end{abstract}

Keywords: dynamo, magnetic buoyancy

\section{Introduction}

From 1990s a type of model has been developed for the solar dynamo known as the flux transport dynamo model (Wang et al., 1991; Choudhuri et al., 1995; Durney, 1995). At the present time, this model seems to be the most promising and satisfactory model for explaining different aspects of the solar cycle (Choudhuri, 2011, 2015; Charbonneau, 2014), although still doubts are sometimes expressed about its validity. The flux transport dynamo model

\footnotetext{
*Corresponding author

Email addresses: arnab@physics.iisc.ernet.in (Arnab Rai Choudhuri), ghazra@physics.iisc.ernet.in (Gopal Hazra)
} 
basically involves the following three processes. (i) The strong toroidal field is produced by the stretching of the poloidal field by differential rotation in the tachocline. (ii) The toroidal field generated in the tachocline gives rise to active regions due to magnetic buoyancy, and the decay of tilted bipolar active regions produces the poloidal field by the Babcock-Leighton mechanism. (iii) The poloidal field produced by the Babcock-Leighton mechanism is advected by the meridional circulation first to high latitudes and then down to the tachocline, while also diffusing down to the tachocline due to turbulent diffusion.

Most of the calculations of the flux transport dynamo model have been based on axisymmetric 2D kinematic mean field equations. It is completely straightforward to include the process (i) within such a formalism - especially because helioseismology gives us the profile of differential rotation. As far as process (iii) is concerned, there are some uncertainties in the nature of the meridional circulation (Hathaway, 2012; Zhao et al., 2013; Schad et al., 2013; Rajaguru and Antia, 2015) as well as in the value of turbulent diffusion (Jiang et al., 2007; Yeates et al., 2008). Although early models assumed a simple one-cell form of meridional circulation, there have been some recent calculations with more complicated meridional circulation (Hazra et al., 2014). However, once we specify the form of the meridional circulation and the turbulent diffusion within the framework of the kinematic model, there is absolutely no uncertainty in the mathematical forms of the terms involved in process (iii). Only in the case of process (ii) involving magnetic buoyancy and the Babcock-Leighton mechanism, there is considerable uncertainty at a fundamental level as to how this process should be included in a $2 \mathrm{D}$ kinematic model. Magnetic buoyancy involves the rise of a tilted flux loop through the convection zone and is an inherently 3D process, which can be included in a 2D dynamo model only through rather crude approximation procedures. Over the years, different groups have proposed different procedures for handling the process (ii). While carrying on calculations with the flux transport dynamo model, we have become aware that these different procedures often give significantly different results and we have tried to understand the physical reasons behind these differences. The obvious question is: which one is the most realistic procedure for treating the process (ii)? This is not an easy question to settle. Different procedures have their own strengths and own weaknesses. We have found that the subtleties involved in modelling the process (ii) are not sufficiently appreciated by the scientific community. Probably a fully satisfactory treatment of process (ii) is not possible within 
the 2D kinematic framework and one has to go beyond 2D. We review the different procedures which had been proposed by different groups and critically examine the aspects of physics which are covered and which are not covered in these different procedures.

\section{The basic equations}

We assume both the mean magnetic field and the mean velocity field to be axisymmetric in $2 \mathrm{D}$ kinematic models. The magnetic field is written as

$$
\mathbf{B}=B(r, \theta) \mathbf{e}_{\phi}+\nabla \times\left[A(r, \theta) \mathbf{e}_{\phi}\right],
$$

where $B(r, \theta)$ is the toroidal component and $A(r, \theta)$ gives the poloidal component. We can write the velocity field as $\mathbf{v}+r \sin \theta \Omega(r, \theta) \mathbf{e}_{\phi}$, where $\Omega(r, \theta)$ is the angular velocity in the interior of the Sun and $\mathbf{v}$ is the velocity of meridional circulation having components in $r$ and $\theta$ directions. Then the main equations telling us how the poloidal and the toroidal fields evolve with time are

$$
\begin{aligned}
\frac{\partial A}{\partial t}+\frac{1}{s}(\mathbf{v} \cdot \nabla)(s A) & =\lambda_{T}\left(\nabla^{2}-\frac{1}{s^{2}}\right) A+S(r, \theta, t) \\
\frac{\partial B}{\partial t}+\frac{1}{r}\left[\frac{\partial}{\partial r}\left(r v_{r} B\right)+\frac{\partial}{\partial \theta}\left(v_{\theta} B\right)\right] & =\lambda_{T}\left(\nabla^{2}-\frac{1}{s^{2}}\right) B+s\left(\mathbf{B}_{p} . \nabla\right) \Omega+\frac{1}{r} \frac{d \lambda_{T}}{d r} \frac{\partial}{\partial r}(r B),
\end{aligned}
$$

where $s=r \sin \theta, \lambda_{T}$ is the turbulent diffusivity and $S(r, \theta, t)$ is the dynamo source term.

The term $s\left(\mathbf{B}_{p} . \nabla\right) \Omega$ in (3) corresponds to process (i) involving the generation of the toroidal field from the poloidal field involving differential rotation. On the other hand, the terms $s^{-1}(\mathbf{v} . \nabla)(s A)$ and $\lambda_{T}\left(\nabla^{2}-1 / s^{2}\right) A$ in (2) correspond to process (iii) involving the evolution of the poloidal field due to the meridional circulation and the turbulent diffusion together. It is the source term $S(r, \theta, t)$ which incorporates the process (ii). Sometimes we have to do some extra things to (2) as well (as described below) in order to include the magnetic buoyancy of the toroidal field $B$.

In the early $\alpha \Omega$ dynamo model postulated by Parker (1955) and Steenbeck et al. (1966), the source term is $S=\alpha B$ (see, for example, Choudhuri (1998), Chapter 16). Here $\alpha$ is a measure of helical turbulence and is usually referred as the $\alpha$-effect. Although the Babcock-Leighton mechanism also can 
be encaptured by a superficially similar $\alpha$-coefficient, its physical interpretation is completely different from that of the $\alpha$-effect, which implies the twisting of the toroidal field. When it was realized that the toroidal field at the bottom of the convection zone is much stronger than what was assumed earlier (Choudhuri and Gilman, 1987; Choudhuri, 1989; D'Silva and Choudhuri, 1993; Fan et al., 1993; Caligari et al., 1995) and the traditional $\alpha$-effect would be suppressed, the Babcock-Leighton mechanism was invoked to take its place, with a similar-looking $\alpha$-coefficient having a different interpretation (Durney, 1997). Since the Babcock-Leighton mechanism primarily takes place near the surface, the $\alpha$-coefficient corresponding to it is usually assumed to be confined near the solar surface. Choudhuri et al. (1995) simply took $S=\alpha B$, with $\alpha$ concentrated at the surface as expected. Even with such a source function which did not include magnetic buoyancy explicitly, they were able to get a periodic solution because of the term

$$
\frac{1}{r}\left[\frac{\partial}{\partial r}\left(r v_{r} B\right)+\frac{\partial}{\partial \theta}\left(v_{\theta} B\right)\right]
$$

in (3), which implied that the toroidal field generated at the tachocline was advected by the meridional circulation to the surface where the BabcockLeighton mechanism operated on it. Küker et al. (2001) also followed this approach.

Although it is possible to construct a Babcock-Leighton dynamo model in this way without explicitly including magnetic buoyancy, this is certainly not very physical or satisfactory. When the toroidal field becomes sufficiently strong, its rise time due to magnetic buoyancy is expected to be much shorter than the advection time by the meridional circulation. So magnetic buoyancy is expected to dominate over such advection and has to be included in the model to make it more realistic. In the next Section, we discuss two popular procedures for incorporating magnetic buoyancy - a non-local procedure and a local procedure. We first discuss how these procedures are used in order to obtain regular dynamo solutions. Then we shall point out in $\S 4$ that we get into added complications when we want to study irregularities of the solar cycle.

\section{Non-local and local treatment of magnetic buoyancy}

The methods of specifying magnetic buoyancy in 2D kinematic models of the flux transport dynamo can be broadly classified into two categories: 
non-local and local. Since the non-local treatment of magnetic buoyancy is somewhat simpler, we discuss that first.

\subsection{Non-local magnetic buoyancy}

The Babcock-Leighton mechanism essentially involves the generation of the poloidal field near the surface from the strong toroidal field at the bottom of the convection zone, which has risen due to magnetic buoyancy. A simple way of incorporating it is to take the source term $S(r, \theta, t)$ in $(2)$ to have the form

$$
S(r, \theta, t)=\alpha(r, \theta) B\left(r=r_{\mathrm{bot}}, \theta, t\right),
$$

in which we usually take $\alpha(r, \theta)$ to be significantly non-zero only near the surface, to ensure that $S(r, \theta, t)$ makes a contribution only near the surface. Also, to get $S(r, \theta, t)$ near the surface, we multiply $\alpha(r, \theta)$ not by the toroidal field $B$ there, but by the toroidal field $B\left(r=r_{\text {bot }}, \theta, t\right)$ at the bottom of the convection zone. Since the rise time due to magnetic buoyancy is small compared to the period of the dynamo, we normally use the same $t$ in $S$ and $B$ without introducing any time delay.

To the best of our knowledge, the method of treating magnetic buoyancy in this way was first proposed by Choudhuri and Dikpati (1999) in their study of the evolution of the solar poloidal field. Afterwards, Dikpati and Charbonneau (1999) adopted it for their flux transport dynamo model. Some other authors have followed this procedure in their dynamo calculations since that time (Charbonneau and Dikpati, 2000; Guerrero and Muñoz, 2004; Charbonneau et al., 2005; Hotta and Yokoyama, 2010).

This method of treating magnetic buoyancy is a simple, robust and stable method. It is found that dynamo models based on this method of treating magnetic buoyancy remain stable on changing the values of basic parameters over wide ranges. However, in spite of its simplicity and attractiveness, this method has the following unphysical features.

(1) We expect the toroidal field to be unstable to magnetic buoyancy only after it has become sufficiently strong. However, in the non-local method of treating buoyancy, even a very weak toroidal field at the bottom of the convection zone starts contributing to the source term in (2).

(2) As a result of buoyant rise from the bottom of the convection zone, the toroidal field at the bottom of the convection keeps getting weaker. The simple method of treating magnetic buoyancy described above does not incorporate this effect and most of the authors who treated magnetic buoyancy in this way did not allow the weakening of the toroidal field due to 
magnetic buoyancy. As we shall see in the next Section, not doing this has serious consequences when we study irregularities of the solar cycle such as the Waldmeier effect.

\subsection{Local magnetic buoyancy}

In this approach, some toroidal magnetic field is transferred from the bottom of the convection zone to the solar surface and then this toroidal field at the surface is expected to produce the poloidal field locally. Magnetic buoyancy is known to be particularly destabilizing within the convection zone (Parker, 1975; Moreno-Insertis, 1983). So, as the toroidal field evolves according to (3), we check at periodic intervals if the toroidal field at any point within the convection zone becomes stronger than a critical value $B_{c}$ and, if so, then some toroidal field from such region is transferred to the surface. It may noted that this procedure introduces a limit to the growth of the dynamo by not allowing $B$ to grow much beyond $B_{c}$. As a result, the dynamo can exhibit non-growing oscillatory solutions even without introducing any kind of quenching. On the other hand, in the non-local procedure described in $\S 3.1$, it is absolutely essential to include some kind of quenching (a quenching of the $\alpha$-coefficient being the most common) to stop the runaway growth of the magnetic field. After the toroidal field is shifted to the top of the convection zone, we have to prescribe some way of generating the poloidal field from it. Two ways of doing this are described in the next two paragraphs.

Local $\alpha$ parameterization. One way is to prescribe the source term $S(r, \theta, t)$ in (2) simply as a product of $\alpha(r, \theta)$ confined around the surface and the toroidal field $B(r, \theta, t)$ there, which has been shifted there from those regions at a bottom of the convection zone where $B$ exceeded $B_{c}$ in a way that ensured the conservation of the toroidal flux (i.e. the amount of toroidal flux deposited near the surface has to equal the amount of toroidal flux removed from the bottom of the convection zone). This method has been followed in several publications from our group (Nandy and Choudhuri, 2001, 2002; Chatteriee et al., 2004; Choudhuri et al., 2005; Chatteriee and Choudhuri, 2006; Choudhuri et al., 2007; Goel and Choudhuri, 2009; Karak and Choudhuri, 2011, 2012, 2013; Hazra et al., 2015).

Durney's double ring method. The ideas of Babcock (1961) and Leighton (1969) were followed more closely by Durney (1995, 1997). Due to the action of the Coriolis force, the rising flux tube gets tilted (D'Silva and Choudhuri, 1993) and produces two sunspots of opposite polarity at slightly different latitudes. In an axisymmetric 2D formulation, we have to average over longitude, 


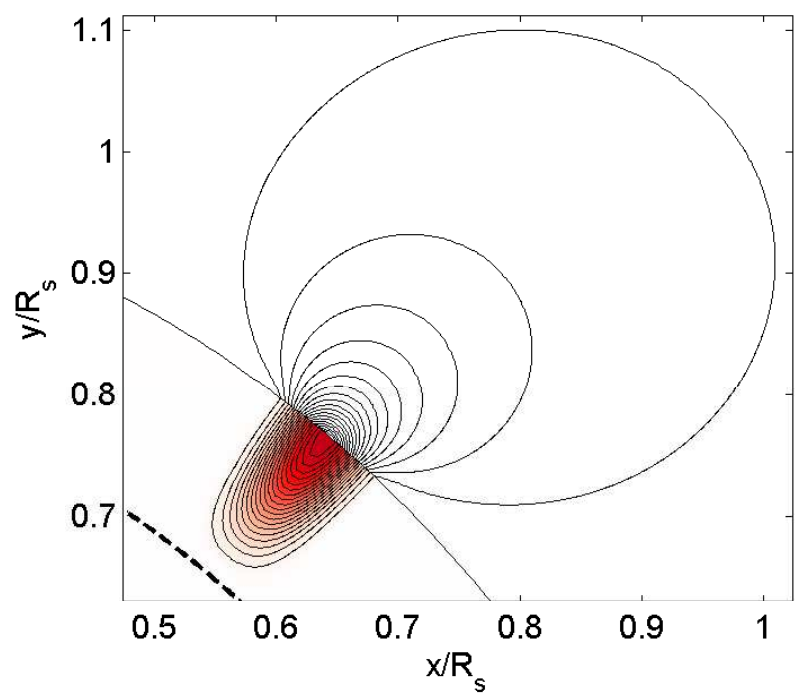

Figure 1: The poloidal field lines of a double ring formed near the surface due to magnetic buoyancy. Taken from Muñoz-Jaramillo et al. (2010).

which gives us two flux rings of opposite sign at two slightly different latitudes. The generation of the poloidal field is prescribed in the following way. Whenever $B$ in some region within the convection exceeds a critical value $B_{c}$, we assume that a part of this $B$ gives rise to the flux ring above the region and we put $A$ appropriate for this flux ring in (2). Here we do not discuss the details of how we find $A$ appropriate for a flux ring, except to mention that some assumption has to be made about the magnetic field structure below and above the flux ring. Presumably the bipolar sunspot pair eventually gets disconnected from the toroidal flux ring at the bottom of the convection zone from which it formed, though it is not clear at the present time how and when this disconnection takes place (Longcope and Choudhuri, 2002). Figure 1 from Muñoz-Jaramillo et al. (2010) shows the typical poloidal field structure assumed by them in their dynamo model with Durney's double ring algorithm.

It is found that the above two methods-local $\alpha$ parameterization and Durney's double ring method - give qualitatively similar results (Nandy and Choudhuri, 2001). However, Muñoz-Jaramillo et al. (2010) have argued that the double ring method is a superior method from the conceptual point of view. 


\subsection{Comparison between the non-local and local methods}

It would have been wonderful if the non-local and local methods of treating magnetic buoyancy gave qualitatively similar results. Unfortunately, that is not the case! When this was first discovered by Choudhuri et al. (2005), it came as a surprise to most of the researchers in the field, though now from hindsight we feel that this should have been an expected result. Figure 2 shows the poloidal field configurations in two dynamo models in which magnetic buoyancy is treated by non-local and local ( $\alpha$ parameterization) methods, but which are identical dynamo models in all other respects. When we use non-local buoyancy, even the weak toroidal fields produced at the high latitude start giving rise to the poloidal field. This causes a multi-lobe structure of the poloidal field in this case, shortening the period of the dynamo. While the model with the local $\alpha$ parameterization gives a period of 14 years, we get a period of barely 6.1 years on using the non-local treatment of magnetic buoyancy.

Because of these big differences, we cannot avoid the question as to which of these two methods is better. We first note that both the methods are gross over-simplifications of a complicated 3D process. In this sense, neither of these two methods can be taken as a realistic depiction of magnetic buoyancy. We believe that the local treatment is a more physical and realistic depiction of what actually happens in the Sun - a conclusion that will be further reinforced from the discussion of the next Section. We have used this method of local treatment in most of the calculations done in our group. Unfortunately, this method is less robust and less stable than the non-local method and seems to give results only within a narrow range of various important dynamo parameters. So, when we want to vary our dynamo parameters over a wide range or when we use unusual parameter specifications (such as a multi-cell meridional circulation), we are often unable to obtain results with the local $\alpha$ parameterization procedure and are forced to use the more robust non-local procedure described in $§ 3.1$ (Karak et al., 2014b; Hazra et al., 2014).

\section{Modelling irregularities of the solar cycle}

The previous section outlined the various ways of treating magnetic buoyancy and pointed out the differences in the periodic dynamo solutions we get with them. Within the last few years, one of the goals of solar dynamo 

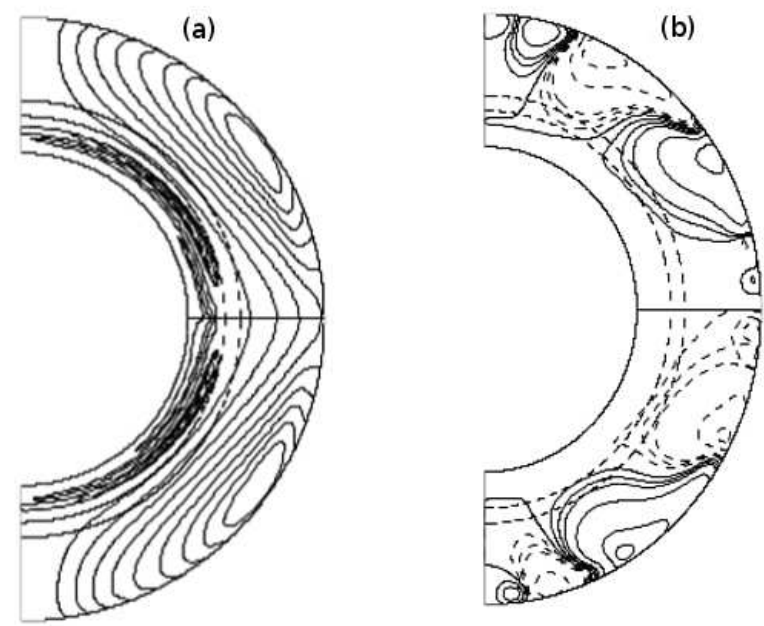

Figure 2: Poloidal field lines at a particular instant of time in two solar dynamo simulations. Both use the same combination of parameters which were used to generate the standard model in $\S 4$ of Chatteriee et al. (2004). The only difference between the two runs is that (a) was generated by using local buoyancy treatment and (b) was generated by using the non-local buoyancy treatment. Whereas (a) is taken from Fig. 14 of Chatteriee et al. (2004), (b) is taken from Fig. 1 of Choudhuri et al. (2005). 
theory has been to model the irregularities of the solar cycle. Only very recently we realized that different formulations of magnetic buoyancy may give radically different results in this important field of study - especially when we consider irregularities of the solar cycle caused by the variations in the meridional circulation.

The duration of the solar cycle becomes more when the meridional circulation slows down (Dikpati and Charbonneau, 1999; Yeates et al., 2008). So it is expected that variations in the meridional circulation will introduce irregularities in the cycle. When this problem is studied with the help of local $\alpha$ parameterization, the theoretical results are broadly in agreement with observational data (Karak, 2010; Karak and Choudhuri, 2011; Choudhuri and Karak, 2012; Karak and Choudhuri, 2013; Hazra et al., 2015). Before explaining how things change on changing the method of treating magnetic buoyancy, let us say a few words about the basic physics of the problem. When a cycle becomes longer due to the slowing of the meridional circulation, two competing effects take place. The differential rotation has more time to generate more toroidal field, trying to make the cycle stronger. On the other hand, diffusion has also more time to act on the magnetic field and tries to make the cycle weaker. Which of these two effects wins over depends on the assumed value of the turbulent diffusion coefficient in the convection zone.

Most of the calculations in our group were done with a value of turbulent diffusion around $10^{12} \mathrm{~cm}^{2} \mathrm{~s}^{-1}$ consistent with mixing length argument (Parker, 1979, p. 629; Jiang et al. 2007). With such a value of turbulent diffusion, the effect of diffusion trying to make the longer cycle weaker wins over the effect of differential rotation trying to make longer cycles stronger. As a result, longer cycles tend to be weaker. This naturally leads to an explanation of the Waldmeier effect that the rise time of the cycle is inversely correlated with the strength of the cycle (Karak and Choudhuri, 2011). On the other hand, several papers from HAO (Dikpati and Charbonneau, 1999; Charbonneau and Dikpati, 2000; Dikpati and Gilman, 2006) used a value of turbulent diffusion about 50 times smaller. With such a value of turbulent diffusion, the effect of differential rotation trying to make longer cycles stronger wins over, giving the opposite of the Waldmeier effect.

The better agreement with observational data on using the higher value of turbulent diffusion clearly indicates that this higher value must be closer to reality. However, we realized only recently that an appropriate handling of magnetic buoyancy is also required to get a match with observational 
data. We basically need diffusion to be dominant so that longer cycles are weaker. When we use a higher value of diffusion and treat magnetic buoyancy through local $\alpha$ parameterization, this happens and we are able to explain effects like the Waldmeier effect beautifully. However, when we use the non-local buoyancy method, we get into trouble even on taking the higher value of diffusion. In our calculations with local $\alpha$ parameterization, toroidal flux is removed from the bottom of the convection zone as a result of magnetic buoyancy. On the other hand, in the non-local buoyancy formulation used in the papers of the HAO group (Dikpati and Charbonneau, 1999; Charbonneau and Dikpati, 2000), the toroidal flux is never depleted as a result of magnetic buoyancy. Because of this, the toroidal field keeps becoming stronger when the cycle is longer and, even with a high value of diffusion, we find that longer cycles tend to be stronger, giving the opposite of the Waldmeier.

Since this aspect of the problem was realized only recently and has not yet been reported in regular journal publications, we present some new results here. Figure 3(a) shows the Waldmeier effect (i.e. the anti-correlation between the rise time and the strength of the cycles) obtained with a dynamo model with a high value of diffusion in the convection zone on using the local $\alpha$ parameterization. If we treat magnetic buoyancy through the non-local method as given by (4), while keeping all other things of the dynamo unchanged, then we get the opposite of the Waldmeier effect, i.e. a correlation rather than an anti-correlation. This is shown in Figure 3(b). To verify that this is indeed caused by not including the depletion of the toroidal field by magnetic buoyancy, if we now modify the non-local method slightly by putting the restriction that the toroidal field is not allowed to grow beyond a limiting value $B_{c}$ (i.e. we put a specification in the code that whenever $B>B_{c}$ we set $B=B_{c}$ ), then we again get back the Waldmeier effect. The result is shown in Figure 3(c) which is obtained by using the non-local buoyancy with the restriction that $B$ is never allowed to grow beyond $B_{c}$. We thus have a rather peculiar situation. Although the non-local magnetic buoyancy method is more robust and works for a wide range of parameters, if this method is used blindly while studying irregularities caused by the variations in the meridional circulation, we are likely to get completely wrong results. This should make it clear that none of the presently used methods of treating magnetic buoyancy is completely satisfactory. Each method has its limitations and we have to keep these limitations in mind when interpreting the results obtained with a particular method. 


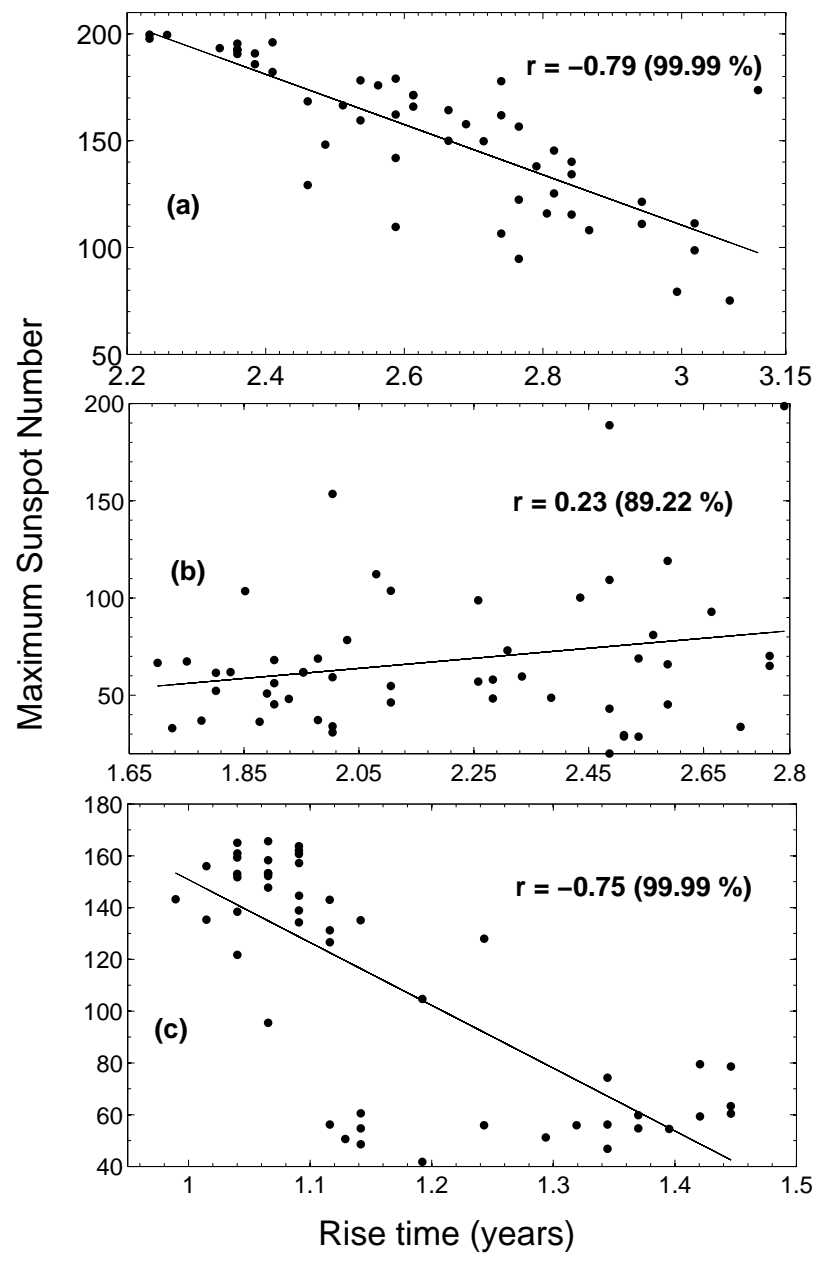

Figure 3: The correlation between the rise and the strength of the solar cycle from a theoretical solar dynamo model using the same combination of parameters as the high diffusivity model presented by Karak and Choudhuri (2011). Only fluctuations in the meridional circulation are included. The panels (a) and (b) are obtained by using respectively the local treatment and the non-local treatment (without depletion in the toroidal flux) of magnetic buoyancy. The last panel (c) obtained by using the non-local treatment of magnetic buoyancy in which the toroidal field is not allowed to grow larger than a critical value $B_{c}$. The panel (a) essentially the same plot as Fig. 4(a) of Karak and Choudhuri (2011) obtained with a different realization of the randomness. 


\section{The latitudinal distribution problem}

So far we have discussed problems of a realistic 2D formulation of magnetic buoyancy, which is an intrinsically 3D process. Now we mention another problem connected possibly with magnetic buoyancy, which is still very poorly understood. In a helioseismic map of differential rotation (see, for example, Schou et al. (1998)), it is clearly seen that differential rotation is concentrated more strongly at high latitudes than at low latitudes - the sign being different at high and low latitudes. Because of this, the generation of the toroidal magnetic field from the poloidal magnetic field is supposed to be more pronounced at the high latitudes. At the low latitudes, the differential rotation present there first has to 'unwind' the toroidal field produced by the differential rotation of the opposite sign at high latitudes and brought to the low latitudes by the equatorward meridional circulation. Only after that, the differential rotation at low latitudes can build up the toroidal field. Hence, when we use the differential rotation discovered by helioseismology, there is a propensity of stronger toroidal field being produced at the high latitudes. If this strong toroidal field is allowed to rise to the surface there due to magnetic buoyancy, then we find sunspots at latitudes higher than where they are seen. The first authors to use helioseismically determined differential rotation already noticed this problem (Dikpati and Charbonneau, 1999; Küker et al., 2001)

Nandy and Choudhuri (2002) proposed a solution to this problem. They suggested that the meridional circulation penetrates slightly below the bottom of the convection zone, where the temperature gradient is stable against convection and magnetic buoyancy is suppressed to a large extent. If this is the case, then the strong toroidal field created in the high-latitude tachocline may be pushed below the bottom of the convection zone and may not be able to rise to the surface due to the suppression of magnetic buoyancy there, thereby inhibiting formation of sunspots at high latitudes. Then the toroidal field would be advected equatorward by the meridional circulation through layers slightly below the bottom of the convection. Then, when the penetrating meridional circulation again enters the convection zone at low latitudes, the toroidal field is brought into the convection zone and magnetic buoyancy again takes over to produce sunspots at low latitudes. This Nandy-

Choudhuri hypothesis became a source of controversy - Gilman and Miesch (2004) arguing that it is not possible for the meridional circulation to penetrate into the stable layers below the bottom of the convection zone, whereas 
Garaud and Brummell (2008) pointed out that such penetration is possible. In many dynamo calculations from our group, we have made the meridional circulation slightly penetrating in order to confine sunspots to low latitudes. As Chakraborty et al. (2009) pointed out, one strong support for the Nandy-Choudhuri hypothesis comes from the observation that torsional oscillations begin at higher latitudes before the start of a sunspot cycle. Since torsional oscillations are presumably driven by the Lorentz force of the dynamo-generated magnetic field, this observation clearly suggests that the strong toroidal field builds up in the high-latitude tachocline a few years before this field is advected to low latitudes and is able to produce sunspots, in accordance with the Nandy-Choudhuri hypothesis.

In a study of the evolution of the poloidal field (in which the dynamo equation was not solved), Dikpati and Choudhuri (1994) mimicked the behaviour of the dynamo by restricting the source term of the poloidal field to low latitudes. Within the last few years, several authors have solved the dynamo equation by artificially restricting the Babcock-Leighton source term to high latitudes in this fashion (Hotta and Yokoyama, 2010; Muñoz-Jaramillo et al., 2010). While this procedure produces nice-looking butterfly diagrams with sunspots restricted to low latitudes, these authors provide no physical justification for this procedure and we are not sure whether this procedure is valid from the point of view of basic physics. In our opinion, we still do not have a proper understanding of why sunspots are restricted only to low latitudes. Although this is an old problem and no significant progress has been made in the last few years, we decided to add this Section because the existence of this problem is nowadays often not appreciated or acknowledged. We should keep this problem in mind and should not sweep it under the rug.

\section{Conclusion}

Although we so far do not have anything that can be called the standard model of the solar dynamo (Choudhuri, 2008), flux transport dynamo models developed by different groups have lots of common features. While there is a controversy on the nature of the meridional circulation at present, it is still not clear whether a serious revision of existing dynamo models will be required. Another uncertainty about the value of turbulent diffusion seems to be resolved now in favour of higher diffusivity (such that the diffusion time scale is a few years) because only with such diffusivity it is possible to model various aspects of cycle irregularities (Choudhuri, 2015). Whether 
turbulent pumping is important for the solar dynamo is another question. However, since the inclusion of turbulent pumping does not change the results of high-diffusion dynamo models too much (Karak and Nandy, 2012), this is presumably not a large source of uncertainty.

As we have discussed here, the biggest uncertainty in the flux transport dynamo at the present time arises from the treatment of magnetic buoyancy. There have been two widely used procedures for treating magnetic buoyancy in 2D kinematic models of the flux transport dynamo: (i) the non-local treatment in which the toroidal field at the bottom of the convection zone is assumed to contribute directly to the generation of the poloidal field, without itself being depleted, and (ii) the local treatment in which a part of the toroidal field from the bottom of the convection zone is shifted to the top whenever it exceeds a critical value. With magnetic buoyancy treated in either of these ways, it is possible to arrange the parameters of the model in such a way that the dynamo solution reproduces various characteristics of the solar cycle. However, for the same set of parameters, we get very different results on using these different treatments of magnetic buoyancy. The nonlocal treatment is more robust and is preferred when we want to study the behaviour of our system over a wide range of parameters. On the other hand, the local treatment with the toroidal field depletion at the bottom of the convection zone allows the proper reproduction of results under irregular situations (such as the Waldmeier effect) and appears the more physically realistic treatment.

This is certainly an unsatisfactory situation and presumably we cannot model magnetic buoyancy sufficiently well if we restrict ourselves only to strictly 2D situations. It may be noted that, in the early studies of magnetic buoyancy based on thin flux tube equation (Spruit, 1981; Choudhuri, 1990), some physical effects could be studied through 2D calculations (Choudhuri and Gilman, 1987; Choudhuri and D'Silva, 1990; D'Silva and Choudhuri, 1991). However, a proper study of the tilt of bipolar sunspot regions (which is responsible for the Babcock-Leighton process) could be carried out only when the calculations were carried out in 3D (D'Silva and Choudhuri, 1993; Fan et al., 1993; Caligari et al., 1995). Essentially, magnetic buoyancy is inherently a 3D process and cannot be included very satisfactorily in 2D kinematic dynamo models. Further advances in 2D kinematic models are unlikely to solve the problems we are facing now.

The ultimate goal of solar dynamo models is to construct fully dynamically consistent 3D models, in which the velocity fields are also calculated 
from the fundamental equations (Karak et al., 2014a). Beyond 2D kinematic models, however, there is an intermediate possibility - 3D kinematic models, in which the mean velocity fields (such as differential rotation and meridional circulation) are supposed to be axisymmetric and are specified (i.e. not calculated from the basic fluid equations), but the magnetic field is treated in a full $3 \mathrm{D}$ fashion and is allowed to evolve non-axisymmetrically so that we are able to model the formation of tilted bipolar sunspots and the Babcock-Leighton process more realistically. The relatively few citations (according to ADS) to the first paper suggesting this approach (Yeates and Muñoz-Jaramillo, 2013) indicate that the importance of this approach is not generally recognized. But we believe that this is going to be the next important step in flux transport dynamo theory beyond 2D kinematic model. Only a few authors have so far presented results with this approach (Miesch and Dikpati, 2014). While this approach, when developed and integrated with a realistic dynamo model, should be more satisfactory than the currently widely used two treatments of magnetic buoyancy in 2D kinematic models. However, a 3D kinematic model is not expected to immediately solve all the problems connected with magnetic buoyancy in the 2D kinematic model. For example, we have discussed in $\S 5$ the problem of why sunspots appear in lower latitudes. This problem will not be solved with the 3D formulation of magnetic buoyancy. Rather, we shall need a better understanding of the physics of the tachocline to address this question. We thus expect a combination of more realistic calculation of magnetic buoyancy coupled with a better understanding of the tachocline to put the flux transport dynamo on a more satisfactory footing.

\section{Acknowledgements}

We thank Bidya Binay Karak for valuable discussions. This work is partly supported by DST through the J.C. Bose Fellowship awarded to ARC. GH thanks organizers of NASA LWS Workshop On Solar Dynamo Frontiers and Flux Emergence Workshop for providing travel support. GH also thanks CSIR india for financial support.

\section{References}

\section{References}

Babcock, H.W., 1961. The Topology of the Sun's Magnetic Field and the 22-YEAR Cycle. Astrophys. J. 133, 572. doi:10.1086/147060. 
Caligari, P., Moreno-Insertis, F., Schüssler, M., 1995. Emerging flux tubes in the solar convection zone. 1: Asymmetry, tilt, and emergence latitude. Astrophys. J. 441, 886-902. doi:10.1086/175410.

Chakraborty, S., Choudhuri, A.R., Chatterjee, P., 2009. Why Does the Sun's Torsional Oscillation Begin before the Sunspot Cycle? Physical Review Letters 102, 041102. doi:10.1103/PhysRevLett.102.041102, arXiv:0907.4842.

Charbonneau, P., 2014. Solar Dynamo Theory. Ann. Rev. Astron. Astrophys. 52, 251-290. doi:10.1146/annurev-astro-081913-040012.

Charbonneau, P., Dikpati, M., 2000. Stochastic Fluctuations in a BabcockLeighton Model of the Solar Cycle. Astrophys. J. 543, 1027-1043. doi:10.1086/317142.

Charbonneau, P., St-Jean, C., Zacharias, P., 2005. Fluctuations in BabcockLeighton Dynamos. I. Period Doubling and Transition to Chaos. Astrophys. J. 619, 613-622. doi:10.1086/426385.

Chatterjee, P., Choudhuri, A.R., 2006. On Magnetic Coupling Between the Two Hemispheres in Solar Dynamo Models. Solar Phys. 239, 29-39. doi:10.1007/s11207-006-0201-6.

Chatterjee, P., Nandy, D., Choudhuri, A.R., 2004. Full-sphere simulations of a circulation-dominated solar dynamo: Exploring the parity issue. Astron. Astrophys. 427, 1019-1030. doi:10.1051/0004-6361:20041199, arXiv:astro-ph/0405027.

Choudhuri, A.R., 1989. The evolution of loop structures in flux rings within the solar convection zone. Solar Phys. 123, 217-239. doi:10.1007/BF00149104.

Choudhuri, A.R., 1990. A correction to Spruit's equation for the dynamics of thin flux tubes. Astron. Astrophys. 239, 335-339.

Choudhuri, A.R., 1998. The physics of fluids and plasmas : an introduction for astrophysicists (Cambridge University Press).

Choudhuri, A.R., 2008. How far are we from a Standard Model of the solar dynamo? Advances in Space Research 41, 868-873. doi:10.1016/j.asr.2007.04.006. 
Choudhuri, A.R., 2011. The origin of the solar magnetic cycle. Pramana 77, 77-96. doi:10.1007/s12043-011-0113-4, arXiv:1103.3385.

Choudhuri, A.R., 2015. A Critical Assessment of the Flux Transport Dynamo. Journal of Astrophysics and Astronomy 36, 5-14. doi:10.1007/s12036-015-9323-3, arXiv:1408.3968.

Choudhuri, A.R., Chatterjee, P., Jiang, J., 2007. Predicting Solar Cycle 24 With a Solar Dynamo Model. Physical Review Letters 98, 131103. doi:10.1103/PhysRevLett.98.131103, arXiv:astro-ph/0701527.

Choudhuri, A.R., Dikpati, M., 1999. On the large-scale diffuse magnetic field of the Sun - II.The Contribution of Active Regions. Solar Phys. 184, 61-76. doi:10.1023/A:1005092601436.

Choudhuri, A.R., D'Silva, S., 1990. Influence of turbulence on rising flux tubes in the solar convection zone. Astron. Astrophys. 239, 326-334.

Choudhuri, A.R., Gilman, P.A., 1987. The influence of the Coriolis force on flux tubes rising through the solar convection zone. Astrophys. J. 316, 788-800. doi:10.1086/165243.

Choudhuri, A.R., Karak, B.B., 2012. Origin of Grand Minima in Sunspot Cycles. Physical Review Letters 109, 171103. doi:10.1103/PhysRevLett.109.171103, arXiv:1208.3947.

Choudhuri, A.R., Nandy, D., Chatterjee, P., 2005. Reply to the Comments of Dikpati et al. Astron. Astrophys. 437, 703-704. doi:10.1051/0004-6361:20052934, arXiv:astro-ph/0505232.

Choudhuri, A.R., Schüssler, M., Dikpati, M., 1995. The solar dynamo with meridional circulation. Astron. Astrophys. 303, L29.

Dikpati, M., Charbonneau, P., 1999. A Babcock-Leighton Flux Transport Dynamo with Solar-like Differential Rotation. Astrophys. J. 518, 508-520. doi:10.1086/307269.

Dikpati, M., Choudhuri, A.R., 1994. The evolution of the Sun's poloidal field. Astron. Astrophys. 291, 975-989. 
Dikpati, M., Gilman, P.A., 2006. Simulating and Predicting Solar Cycles Using a Flux-Transport Dynamo. Astrophys. J. 649, 498-514. doi:10.1086/506314.

D'Silva, S., Choudhuri, A.R., 1993. A theoretical model for tilts of bipolar magnetic regions. Astron. Astrophys. 272, 621.

D'Silva, S.Z., Choudhuri, A.R., 1991. The effect of Kelvin-Helmholtz instability on rising flux tubes in the convection zone. Solar Phys. 136, 201-219. doi:10.1007/BF00146531.

Durney, B.R., 1995. On a Babcock-Leighton dynamo model with a deepseated generating layer for the toroidal magnetic field. Solar Phys. 160, 213-235. doi $10.1007 / \mathrm{BF} 00732805$.

Durney, B.R., 1997. On a Babcock-Leighton Solar Dynamo Model with a Deep-seated Generating Layer for the Toroidal Magnetic Field. IV. Astrophys. J. 486, 1065-1077.

Fan, Y., Fisher, G.H., Deluca, E.E., 1993. The origin of morphological asymmetries in bipolar active regions. Astrophys. J. 405, 390-401. doi:10.1086/172370.

Garaud, P., Brummell, N.H., 2008. On the Penetration of Meridional Circulation below the Solar Convection Zone. Astrophys. J. 674, 498-510. doi:10.1086/524837, arXiv:0708.0258.

Gilman, P.A., Miesch, M.S., 2004. Limits to Penetration of Meridional Circulation below the Solar Convection Zone. Astrophys. J. 611, 568-574. doi:10.1086/421899.

Goel, A., Choudhuri, A.R., 2009. The hemispheric asymmetry of solar activity during the last century and the solar dynamo. Research in Astronomy and Astrophysics 9, 115-126. doi:10.1088/1674-4527/9/1/010, arXiv:0712.3988.

Guerrero, G.A., Muñoz, J.D., 2004. Kinematic solar dynamo models with a deep meridional flow. Mon. Not. Roy. Astron. Soc. 350, 317-322. doi:10.1111/j.1365-2966.2004.07655.x, arXiv:astro-ph/0402097. 
Hathaway, D.H., 2012. Supergranules as Probes of the Sun's Meridional Circulation. Astrophys. J. 760, 84. doi:10.1088/0004-637X/760/1/84, arXiv: 1210.3343 .

Hazra, G., Karak, B.B., Banerjee, D., Choudhuri, A.R., 2015. Correlation Between Decay Rate and Amplitude of Solar Cycles as Revealed from Observations and Dynamo Theory. Solar Phys. 290, 1851-1870. doi:10.1007/s11207-015-0718-8, arXiv:1410.8641.

Hazra, G., Karak, B.B., Choudhuri, A.R., 2014. Is a Deep One-cell Meridional Circulation Essential for the Flux Transport Solar Dynamo? Astrophys. J. 782, 93. doi:10.1088/0004-637X/782/2/93, arXiv:1309.2838.

Hotta, H., Yokoyama, T., 2010. Solar Parity Issue with Fluxtransport Dynamo. Astrophys. J. Lett. 714, L308-L312. doi:10.1088/2041-8205/714/2/L308, arXiv:1004.2088.

Jiang, J., Chatterjee, P., Choudhuri, A.R., 2007. Solar activity forecast with a dynamo model. Mon. Not. Roy. Astron. Soc. 381, 1527-1542. doi:10.1111/j.1365-2966.2007.12267.x, arXiv:0707.2258,

Karak, B.B., 2010. Importance of Meridional Circulation in Flux Transport Dynamo: The Possibility of a Maunder-like Grand Minimum. Astrophys. J. 724, 1021-1029. doi:10.1088/0004-637X/724/2/1021, arXiv:1009.2479.

Karak, B.B., Choudhuri, A.R., 2011. The Waldmeier effect and the flux transport solar dynamo. Mon. Not. Roy. Astron. Soc. 410, 1503-1512. doi:10.1111/j.1365-2966.2010.17531.x, arXiv:1008.0824,

Karak, B.B., Choudhuri, A.R., 2012. Quenching of Meridional Circulation in Flux Transport Dynamo Models. Solar Phys. 278, 137-148. doi:10.1007/s11207-012-0142-2, arXiv:1111.1540,

Karak, B.B., Choudhuri, A.R., 2013. Studies of grand minima in sunspot cycles by using a flux transport solar dynamo model. Research in Astronomy and Astrophysics 13, 1339-1357. doi:10.1088/1674-4527/13/11/005, arXiv:1306.5438. 
Karak, B.B., Jiang, J., Miesch, M.S., Charbonneau, P., Choudhuri, A.R., 2014a. Flux Transport Dynamos: From Kinematics to Dynamics. Space Sci. Rev. 186, 561-602. doi 10.1007/s11214-014-0099-6.

Karak, B.B., Kitchatinov, L.L., Choudhuri, A.R., 2014b. A Dynamo Model of Magnetic Activity in Solar-like Stars with Different Rotational Velocities. Astrophys. J. 791, 59. doi:10.1088/0004-637X/791/1/59, arXiv:1402.1874.

Karak, B.B., Nandy, D., 2012. Turbulent Pumping of Magnetic Flux Reduces Solar Cycle Memory and thus Impacts Predictability of the Sun's Activity. Astrophys. J. Lett. 761, L13. doi:10.1088/2041-8205/761/1/L13, arXiv:1206.2106.

Küker, M., Rüdiger, G., Schultz, M., 2001. Circulation-dominated solar shell dynamo models with positive alpha-effect. Astron. Astrophys. 374, 301308. doi:10.1051/0004-6361:20010686.

Leighton, R.B., 1969. A Magneto-Kinematic Model of the Solar Cycle. Astrophys. J. 156, 1. doi $10.1086 / 149943$.

Longcope, D., Choudhuri, A.R., 2002. The Orientational Relaxation of Bipolar Active Regions. Solar Phys. 205, 63-92. doi:10.1023/A: 1013896013842 .

Miesch, M.S., Dikpati, M., 2014. A Three-dimensional BabcockLeighton Solar Dynamo Model. Astrophys. J. Lett. 785, L8. doi:10.1088/2041-8205/785/1/L8, arXiv:1401.6557.

Moreno-Insertis, F., 1983. Rise times of horizontal magnetic flux tubes in the convection zone of the sun. Astron. Astrophys. 122, 241-250.

Muñoz-Jaramillo, A., Nandy, D., Martens, P.C.H., Yeates, A.R., 2010. A Double-ring Algorithm for Modeling Solar Active Regions: Unifying Kinematic Dynamo Models and Surface Flux-transport Simulations. Astrophys. J. Lett. 720, L20-L25. doi:10.1088/2041-8205/720/1/L20, arXiv: 1007.1262 .

Nandy, D., Choudhuri, A.R., 2001. Toward a Mean Field Formulation of the Babcock-Leighton Type Solar Dynamo. I. $\alpha$-Coefficient versus Durney's 
Double-Ring Approach. Astrophys. J. 551, 576-585. doi:10.1086/320057, arXiv:astro-ph/0107466.

Nandy, D., Choudhuri, A.R., 2002. Explaining the Latitudinal Distribution of Sunspots with Deep Meridional Flow. Science 296, 1671-1673. doi:10.1126/science.1070955.

Parker, E.N., 1955. Hydromagnetic Dynamo Models. Astrophys. J. 122, 293. doi:10.1086/146087.

Parker, E.N., 1975. The generation of magnetic fields in astrophysical bodies. X - Magnetic buoyancy and the solar dynamo. Astrophys. J. 198, 205-209. doi:10.1086/153593.

Rajaguru, S.P., Antia, H.M., 2015. Meridional circulation in the solar convection zone: time-distance helioseismic inferences from four years of HMI/SDO observations. ArXiv e-prints arXiv:1510.01843.

Schad, A., Timmer, J., Roth, M., 2013. Global Helioseismic Evidence for a Deeply Penetrating Solar Meridional Flow Consisting of Multiple Flow Cells. Astrophys. J. Lett. 778, L38. doi:10.1088/2041-8205/778/2/L38, arXiv:1311.7623.

Schou, J., Antia, H.M., Basu, S., Bogart, R.S., Bush, R.I., Chitre, S.M., Christensen-Dalsgaard, J., Di Mauro, M.P., Dziembowski, W.A., EffDarwich, A., Gough, D.O., Haber, D.A., Hoeksema, J.T., Howe, R., Korzennik, S.G., Kosovichev, A.G., Larsen, R.M., Pijpers, F.P., Scherrer, P.H., Sekii, T., Tarbell, T.D., Title, A.M., Thompson, M.J., Toomre, J., 1998. Helioseismic Studies of Differential Rotation in the Solar Envelope by the Solar Oscillations Investigation Using the Michelson Doppler Imager. Astrophys. J. 505, 390-417. doi:10.1086/306146.

Spruit, H.C., 1981. Equations for thin flux tubes in ideal MHD. Astron. Astrophys. 102, 129-133.

Steenbeck, M., Krause, F., Rädler, K.H., 1966. Berechnung der mittleren Lorentz-Feldstärke v X B für ein elektrisch leitendes Medium in turbulenter, durch Coriolis-Kräfte beeinflußter Bewegung. Zeitschrift Naturforschung Teil A 21, 369. 
Wang, Y.M., Sheeley, Jr., N.R., Nash, A.G., 1991. A new solar cycle model including meridional circulation. Astrophys. J. 383, 431-442. doi:10.1086/170800.

Yeates, A.R., Muñoz-Jaramillo, A., 2013. Kinematic active region formation in a three-dimensional solar dynamo model. Mon. Not. Roy. Astron. Soc. 436, 3366-3379. doi:10.1093/mnras/stt1818, arXiv:1309.6342.

Yeates, A.R., Nandy, D., Mackay, D.H., 2008. Exploring the Physical Basis of Solar Cycle Predictions: Flux Transport Dynamics and Persistence of Memory in Advection- versus Diffusion-dominated Solar Convection Zones. Astrophys. J. 673, 544-556. doi:10.1086/524352, arXiv:0709.1046.

Zhao, J., Bogart, R.S., Kosovichev, A.G., Duvall, Jr., T.L., Hartlep, T., 2013. Detection of Equatorward Meridional Flow and Evidence of Doublecell Meridional Circulation inside the Sun. Astrophys. J. Lett. 774, L29. doi:10.1088/2041-8205/774/2/L29, arXiv:1307.8422. 